\title{
Eye Movement Desensitization Reprocessing for Children and Adolescents With Posttraumatic Stress Disorder: A Systematic Narrative Review
}

\author{
Ian G. Barron \\ Caitlin Bourgaize \\ University of Massachusetts, Amherst, Massachusetts \\ Daniela Lempertz \\ Child and Adolescent Psychotherapist, Neuwied, Germany \\ Colleen Swinden \\ University of Chester University, United Kingdom \\ Susan Darker-Smith \\ Shropshire Counselling Service, Shropshire, England
}

\begin{abstract}
There is currently a limited number of studies into the efficacy of eye movement desensitization reprocessing (EMDR) therapy with children and adolescents with posttraumatic stress disorder (PTSD). The current study utilizes a systematic narrative review of methodologies and findings of previous literature reviews and meta-analyses as well as analyzing randomized control trials (RCTs) conducted from 2002 to 2018. Following initial scoping of the extent of studies, two systematic literature searches were conducted, firstly for literature reviews and secondly for recent RCTs. Nine databases were utilized. Eight reviews and seven RCTs were identified and analyzed for quality of methodology and outcome as measured by impact on PTSD symptoms. EMDR was found to be efficacious in reducing children's PTSD symptoms compared to waitlist conditions, with similar outcomes to cognitive behavior therapy (CBT). EMDR was effective with both single-event trauma as well as cumulative trauma such as sexual abuse. EMDR was equally effective with girls and boys as well as children from different cultures. EMDR achieved medium to large effect sizes. Reductions in PTSD were maintained at 2-, 3-, 6-, and 12-month follow-up. In conclusion, EMDR was consistently found to be an efficacious treatment for children with PTSD. Recommendations are made for future practice and research.
\end{abstract}

Keywords: literature review; randomized control trial; eye movement desensitization reprocessing; posttraumatic stress disorder; children

$\mathbf{E}$ ye movement desensitization and reprocessing (EMDR) therapy was developed by Shapiro for adults who had experienced adverse life events (Shapiro, 1989). Since this time EMDR has been extensively researched with adults and has demonstrated efficacy for the treatment of the effects of adverse life events, mainly posttraumatic stress disorder (PTSD). The World Health Organization and the International Society for Traumatic Stress Studies (ISTSS) recommend EMDR therapy as a first line treatment for PTSD in adults, adolescents, and children (ISTSS, 2018; WHO, 2013). The National Institute for Health and Care Excellence (NICE, 2018), however, recommends that EMDR is not used before 3 months post-trauma and then it should be considered for children and young people aged 7-17 years with a 
diagnosis of PTSD or clinically important symptoms of PTSD who have presented more than 3 months after a traumatic event only if they do not respond to or engage with trauma-focused cognitive behavioral therapy (TF-CBT) (NICE, 2018). NICE concluded that

there was limited evidence for eye movement desensitization and reprocessing (EMDR) suggesting possible benefits on PTSD symptoms in children older than 7 years. However, EMDR was found to be less clinically effective and cost effective than all individual trauma-focused CBT interventions. On this basis, the committee decided it should be considered only if children do not respond to or engage with traumafocused CBT. (NICE, 2018, p. 34)

In short, the NICE guidance raises serious questions about the efficacy of EMDR with children and the methodological rigor and bias of EMDR empirical studies.

Paradoxically, the NICE review was limited in itself. While the review considered 11 studies, they excluded 8 of the 11, and their review of EMDR therapy included only 3 randomized control trials (RCTs) (Ahmad \& Sundelin-Wahlsten, 2008; De Roos et al., 2017; Soberman, Greenwald, \& Rule, 2002). Their evaluation omitted Diehle, Opmeer, Boer, Mannarino, and Lindauer (2015) and they overlooked Chemtob, Nakashima, and Carlson (2002); De Roos et al. (2011); and Jaberghaderi, Greenwald, Rubin, Zand, and Dolatabadi (2004). The current review, by contrast, includes 4 important studies. As Dominguez and Lee recognize in their article on treatment guidelines in this special issue, there is

a difference between NICE and ISTSS guidelines with respect to the recommendations for children. While it is clear that some studies should have been included in the NICE guidelines and that this would likely have resulted in a higher recommendation equivalent to ISTSS; the fact that two or three missing studies impacts the analysis underscores the importance of further trials in this area. (p. 257)

Further evidence is also needed that supports or challenges the theoretical constructs that underpin EMDR. The theoretical construct that underpins EMDR therapy posits the existence of an information processing system that naturally assimilates new experiences into already existing memory networks (Shapiro, 2018). Shapiro's (2001) adaptive information processing (AIP) model holds that the primary source of pathology is the presence of memories of adverse life events that have been insufficiently processed, and subsequently, stored in a statespecific form, within their own neural network, which do not link to other memory networks holding adaptive information (Shapiro, 2018). While the EMDR practitioner community support such an understanding, this is not shared universally by other trauma recovery perspectives, such as memory reconsolidation (Nader, 2003).

In line with AIP, the goal of the eight phases of EMDR therapy is to facilitate the processing and adaption of these memories. EMDR therapy's eight-phase treatment approach occurs in a systematic manner: (a) client history and treatment planning; (b) preparation, providing the client with information about the EMDR process and resourcing as needed; (c) assessment of the EMDR target memory; (d) desensitization using bilateral stimulation; (e) installation of the client's chosen positive cognition; (f) the client performs an imaginary body scan, and any somatic feelings are then targeted for reprocessing; (g) closure of complete or incomplete sessions, and preparation for sessions to end; and $(\mathrm{h})$ reevaluation of the target at the subsequent session (Shapiro, 2018). Adaptation of the protocol, however, is recommended in different ways for children at different periods of development (Tinker \& Wilson, 1999).

The first published reports of using EMDR therapy for treating children with PTSD were case studies. Shapiro (1991) and Mendoza-Weitman (1992) each described successful treatment of a single child. Greenwald (1995) was the first to show preliminary evidence of the efficacy of EMDR in treating children with posttraumatic stress reactions. Greenwald conducted a case series with five children who suffered psychological consequences after experiencing hurricane Andrew in Florida, USA. The children, age 4-11, received two sessions of 20-90 minutes duration. Semi-structured interviews and the Problem Rating Scale (PBS) were administered to mothers prior to and following treatment. Posttreatment, all children were reported to have returned to pretrauma levels of functioning.

In contrast to adults, when young children encounter adverse or traumatic life events, their brains are still developing (De Bellis \& Zisk, 2014). Traumatic events can, therefore, have an impact on neurological development, in addition to affecting the way future life experiences are perceived, stored, and in turn impact upon other areas of social, emotional, 
academic development (De Bellis \& Zisk, 2014). Children's extensive reactions to adverse life events can include clinical and sub-clinical symptoms of presentations such as PTSD, separation anxiety, fear of the dark, sleep disturbances, anxiety, depression, phobias, regressive behavior, and social behavior problems (Cook, 2005; Van der Kolk, 2005). Children experiencing adverse life events do not always meet the PTSD diagnostic criteria (Jonkman, Verlinden, Bolle, Boer, \& Lindauer, 2013). Indeed, Jonkman et al. (2013) found that children with more severe traumatization presented with fewer PTSD symptoms. This is a very important issue as children who do not meet the criteria may be excluded from treatment for traumatic experiences.

With adverse life events having the potential to create pervasive long-lasting disruptions to their psychological and social development, children and adolescents need timely and effective treatment (ISTSS, 2019). There have been a number of studies which have demonstrated the effective use of EMDR to treat trauma in children and adolescents. In the treatment of PTSD in children, an adapted EMDR protocol is used, with age and developmental modifications (Adler-Tapia \& Settle, 2008; Greenwald, 1999; Lovett, 2007; Shapiro, Kaslow, \& Maxfield, 2007; Tinker \& Wilson, 1999). However, much of the research regarding the use of EMDR with children and adolescents involves case studies and subclinical populations.

There is currently only a small body of RCTs that examines the efficacy of EMDR with children and adolescents diagnosed with PTSD. In seeking to respond to recent NICE guidance, the aim of this article is to provide a systematic narrative review of methodologies and findings of previous literature reviews, meta-analysis, and recent RCTs that have examined the treatment of children and adolescents diagnosed with PTSD and treated with EMDR therapy, inclusive of studies omitted from NICE (2018).

\section{Methods}

The search methodology was a replication of Wilson et al. (2018) literature review used in assessing the efficacy of EMDR for adults with PTSD. The current systematic review focused on the efficacy of EMDR with children and adolescents who presented with PTSD symptoms. The purpose of replicating Wilson and colleague's methodology was to enable a comparison of rigor and outcome of child and adolescent studies with adult EMDR efficacy literature. Three phases were utilized in the search for studies. An initial phase in the literature search involved a scoping review of literature to ensure there were sufficient child and adolescent studies to warrant a review of the literature at this time. Following this (see Figure 1) a systematic search of systematic reviews of literature and metaanalyses on the efficacy of EMDR with children with PTSD was conducted. To ensure the current review included a sufficient number of rigorous studies for analysis, a third phase involved a systematic search of all randomized control studies since 2002 (see Figure 2).

The search terms used in the current review were eye movement desensitization reprocessing; EMDR; posttraumatic stress; PTSD; systematic review; metaanalysis; randomized control trial; and RCT. The nine databases searched through the University of Massachusetts library's online subscription included Web of Science, Medline, Cochrane Library, ASSIA, PubMed, PsycINFO, Science Direct, CINAHL, and Psych Articles. Inclusion criteria involved studies in English (UK and American spellings) that focused on efficacy of EMDR with children and adolescents who presented with PTSD symptoms. Inclusion and exclusion criteria mirrored the adult review (Wilson et al., 2018). Studies were excluded that were not written in English, did not use a randomized control design, were not peer reviewed, or did not evaluate EMDR studies and include children and adolescents with PTSD. Studies where participants were exclusively adults were excluded from the current review.

As with Wilson et al. (2018), the current study utilized the Critical Appraisal Program Tool (CASP, 2017a, 2017b) to assess the quality of the child and adolescent studies. Appraisal included focus and importance, randomization and matching of participants, dropout rates, blind to treatment, effect size, costbenefit, and impact. Similar to NICE (2018), most studies rated "low" quality. Articles were initially reviewed against the inclusion and exclusion criteria using the article's title and abstract. Articles meeting the inclusion criteria were then read in full. References within articles were searched to ensure thoroughness and avoid articles meeting the criteria being omitted.

\section{Results}

The initial scoping of EMDR studies and reviews of literature indicated the viability of progressing with the systematic search of the literature. In the first systematic search of literature reviews and metaanalyses, 135 articles were removed following the initial title and abstract search. Three were duplicates and 


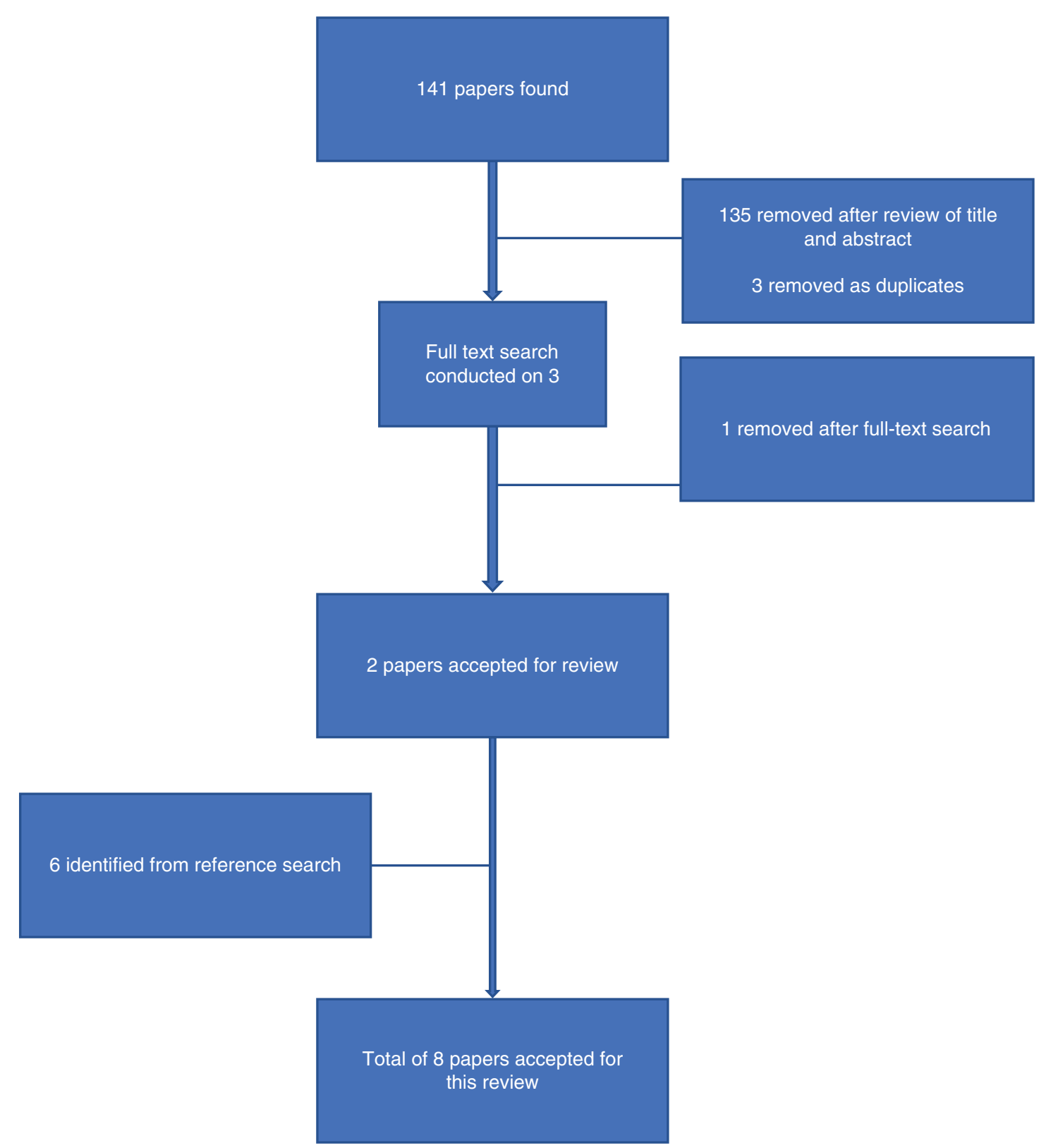

FIGURE 1. EMDR and posttraumatic stress disorder (PTSD) in children: Flow charts. First search = systematic reviews.

$n=3$ were identified through the reference and citation search, which led to no further articles being included. In the second search, this time of RCTs, $n=113$ articles were removed after the initial title and abstract search, two were removed as duplicates, and $n=9$ were identified from a search of articles' references.

\section{Characteristics of Reviews}

Eight systematic reviews were identified from 2009 to 2018. All were conducted by differing groups of authors (see Table 1) and as such avoided author bias. Although all reviewed the efficacy of EMDR in addressing PTSD in children and adolescents, the purpose of the articles differed. Four reviews focused specifically on EMDR (Adler-Tapia \& Settle, 2009; Greyber, Dulmus, \& Cristalli, 2012; Moreno-Alcazar et al., 2017; Rodenburg, Benjamin, De Roos, Meijer, \& Stams, 2009); one addressed the comparison of EMDR with CBT (Khan et al., 2018); one evaluated EMDR, CBT, and KidNET (Brown et al., 2017); and two reviews looked at specific areas of interest (Beer, 2018; Chen et al., 2018). Five reviews focused only on RCTs (Chen et al., 2018; Greyber et al., 2012; Khan et al., 2018; Moreno-Alcazar et al., 2017; Rodenberg et al., 2009), while three summarized RCTs, case studies, and or other methodologies 


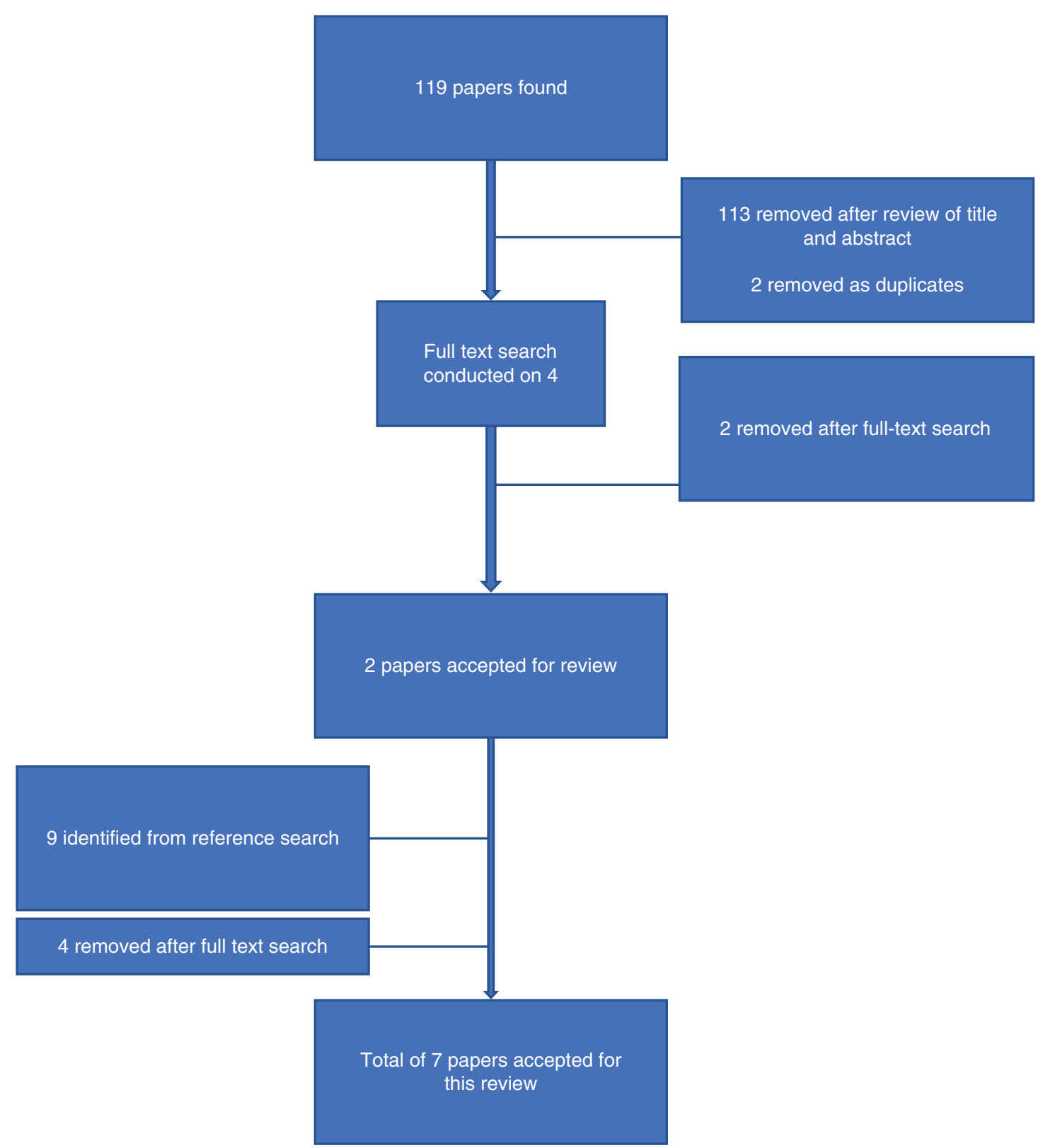

FIGURE 2. EMDR and posttraumatic stress disorder (PTSD) in children: Flow charts. Second search = randomized control trials.

(Adler-Tapia \& Settle, 2009; Beer, 2018; Brown et al., 2017).

Greyber et al. (2012) and Beer (2018) were the only reviews not to include a meta-analysis. The number of studies included in the reviews ranged from 5 to 36. The four reviews which focused only on EMDR therapy evaluated 19 or fewer studies (Adler-Tapia \& Settle, 2009; Greyber et al., 2012; Moreno-Alcazar et al., 2017; Rodenburget al., 2009), with less than 691 total participants. The most recent review of KidNET, TFCBT, and EMDR treatments had an impressive 3,541 participants across the three treatments (Brown et al., 2017).
Two of the reviews failed to examine the quality of the studies reviewed (Brown et al., 2017; Rodenburg et al., 2009), whereas other reviews used differing measures, making it difficult to compare the quality of studies across reviews. Greyber et al. (2012) and Beer (2018) omitted measuring the homogeneity of the populations under study. Again Greyber et al. was the only review not to discuss effect size, although this omission was recognized by the authors. Finally, none of the reviews included the location of where studies were conducted, and in turn there was no examination of the potential relevance of culture and context. 


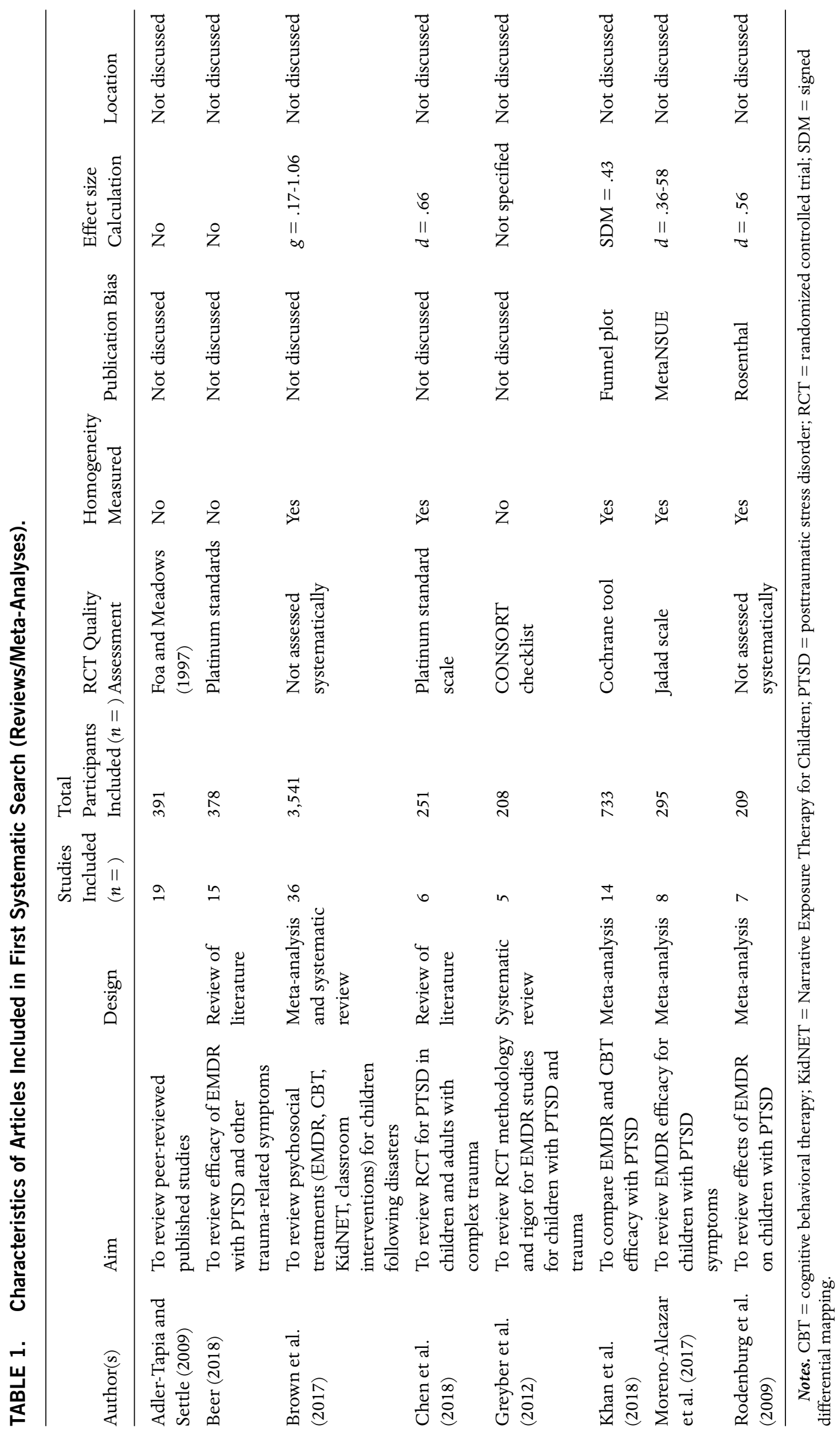




\section{Characteristics of RCTs}

Seven RCTs were identified (see Table 1). An eighth publication was omitted (Ahmad, Bo, \& SundelinWahlsten, 2007) due to errors in statistical analysis and because the study was republished a year later (Ahmad \& Sundelin-Wahlsten, 2008). De Roos was the only principle investigator who led more than one RCT (De Roos et al., 2011; De Roos et al., 2017). See Table 2. All other studies were led by different researchers. Two of the studies failed to report the location of the study (Ahmad \& Sundelin-Wahlsten, 2008; Soberman et al., 2002). The majority of studies $(n=3)$ were conducted in the Netherlands (De Roos, 2011, 2017; Diehle et al. (2015), one was in Iran (Jaberghaderi et al., 2004), and one in Hawaii (Chemtob et al., 2002).

Only four studies conducted diagnostic assessments of participants (Ahmad \& Sundelin-Wahlsten, 2008; Chemtob et al., 2002; De Roos et al., 2017; Diehle et al., 2015). Symptom wise, all studies focused on the efficacy of EMDR on PTSD symptoms. De Roos et al. (2017) and Diehle et al. (2015) also assessed loss of PTSD diagnosis. Some studies measured comorbid symptoms including depression and anxiety (Chemtob et al., 2002; De Roos et al. 2011; De Roos et al. 2017; Diehle et al., 2015) and Soberman et al. (2002) looked at boys' behavioral difficulties. Types of traumas included the sexual abuse of girls ( Jaberghaderi et al., 2004), single trauma (Chemtob et al., 2002; De Roos et al., 2011), disasters (Chem tob et al., 2002; De Roos, 2017), and "psychosocially exposed conditions" (Ahmad \& Sundelin-Wahlsten, 2008). Three RCTs compared EMDR therapy to wait list (Ahmad \& Sundelin-Wahlsten, 2008; Chemtob et al., 2002; Soberman et al., 2002) and four compared the outcomes of CBT and EMDR (De Roos et al., 2011; De Roos et al., 2017; Diehle et al., 2015; Jaberghaderi et al., 2004).

The number of participants in studies ranged from 32 to 103 , with ages ranging from 4 to 18 years old. Children younger than 4 years of age have therefore not been included in EMDR efficacy studies. Five studies covered a wide age range and included both children and adolescents, with only one study (Chemtob et al., 2002) specifically focusing on children (aged 6-12) and one study on adolescent (aged 12-13) girls (Jaberghaderi et al., 2004). Six of the seven studies used a blind protocol where the assessor was not aware of the participants' pretreatment group allocation. Chemtob et al. (2002) was the exception where this was not discussed. Although only two of the studies reported on effect size analysis (De Roos et al., 2017; Diehle et al., 2015), five reported effect sizes;
Ahmad and Sundelin-Wahlsten (2008) and Soberman et al. (2002) were the exceptions. Dropout rates were reported in six of the seven RCTs, with Chemtob et al. (2002) omitting to do so. Dropout rates varied from $2 \%$ (De Roos et al., 2017) to 36\% (Ahmad \& Sundelin-Wahlsten, 2008). Nearly all studies used multiple standardized measures except Ahmad and Sundelin-Wahlsten (2008) enabling comparison across trauma-related symptoms and increasing reliability in the findings.

Number of sessions ranged from 3 (Chemtob et al., 2002; Soberman et al., 2002) to 8 (Ahmad et al., 2008; Diehle et al., 2015;), (Jaberghaderi et al., 2004). Stabilization sessions were therefore minimal. Sessions were mostly reported as 45 minutes duration with minimal homework where mentioned. Five studies did not report family involvement in therapy, although De Roos et al. (2011) and Diehle et al. (2015) included parents in parts of the sessions. All studies conducted pretest and posttest measures to assess the short-term gains of participants. Most studies administered follow-up measures, however, this ranged from 2-week (Jaberghaderi et al., 2004) to 12-month followup (De Roos et al., 2017). There was an inconsistency in follow-up timescales across studies, with 2-month (Ahmad et al., 2007; Ahmad \& Sundelin-Wahlsten, 2008; Diehle et al., 2015; Soberman et al., 2002), 3-month (De Roos et al. (2011, 2017), 4-month, and 6month (Chemtob et al., 2002) follow-up periods.

\section{Analysis of Outcomes}

\section{Effect Sizes Reported in Review Articles}

Three reviews reported effect size as cohen's $d$. All reported medium effect sizes. Moreno-Alcazar et al. (2017) reported individual studies ranges from $d=.36$ to .58 ; Rodenburg et al. (2009) found $d=.56$ and Chen et al. (2018) found the largest effect size of $d=.66$. Khan et al. (2018) reported a medium effect size as signed differential mapping $(S D M=.43)$ and Brown et al. (2017) reported small to large effect sizes across studies using Hedges' $g(g=.17-1.06)$. Three reviews failed to specify effect sizes (Adler-Tapia $\&$ Settle, 2009; Beer, 2018; Greyber et al., 2012).

In comparing EMDR with CBT and KidNET (Brown et al., 2017), all therapies demonstrated significant reductions in PTSD resulting from natural and man-made disasters. No significant differences, however, were found between EMDR, CBT, and KidNET. Interestingly, effects were moderated by type of profession (higher level of training leading to higher effect sizes). 


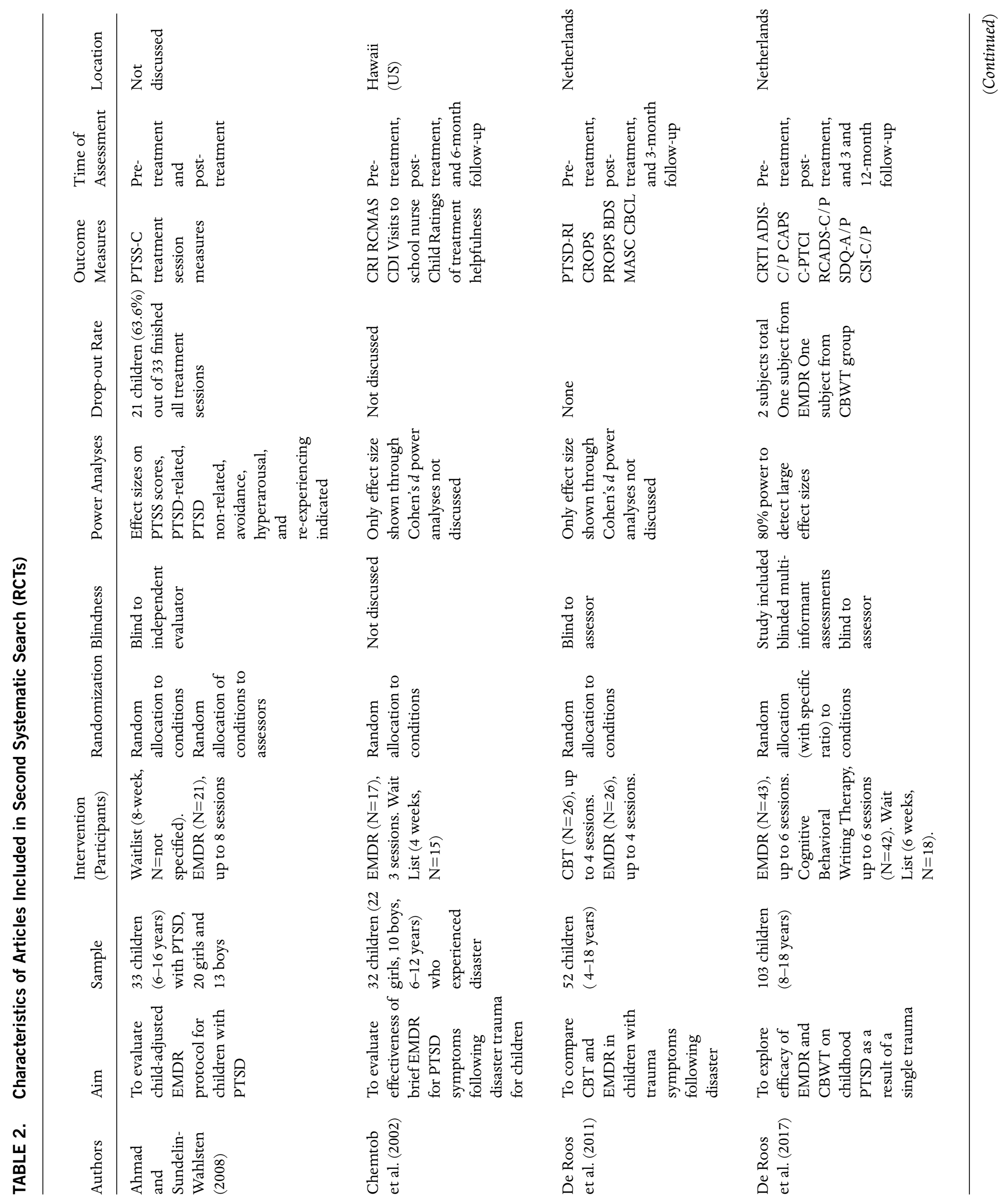




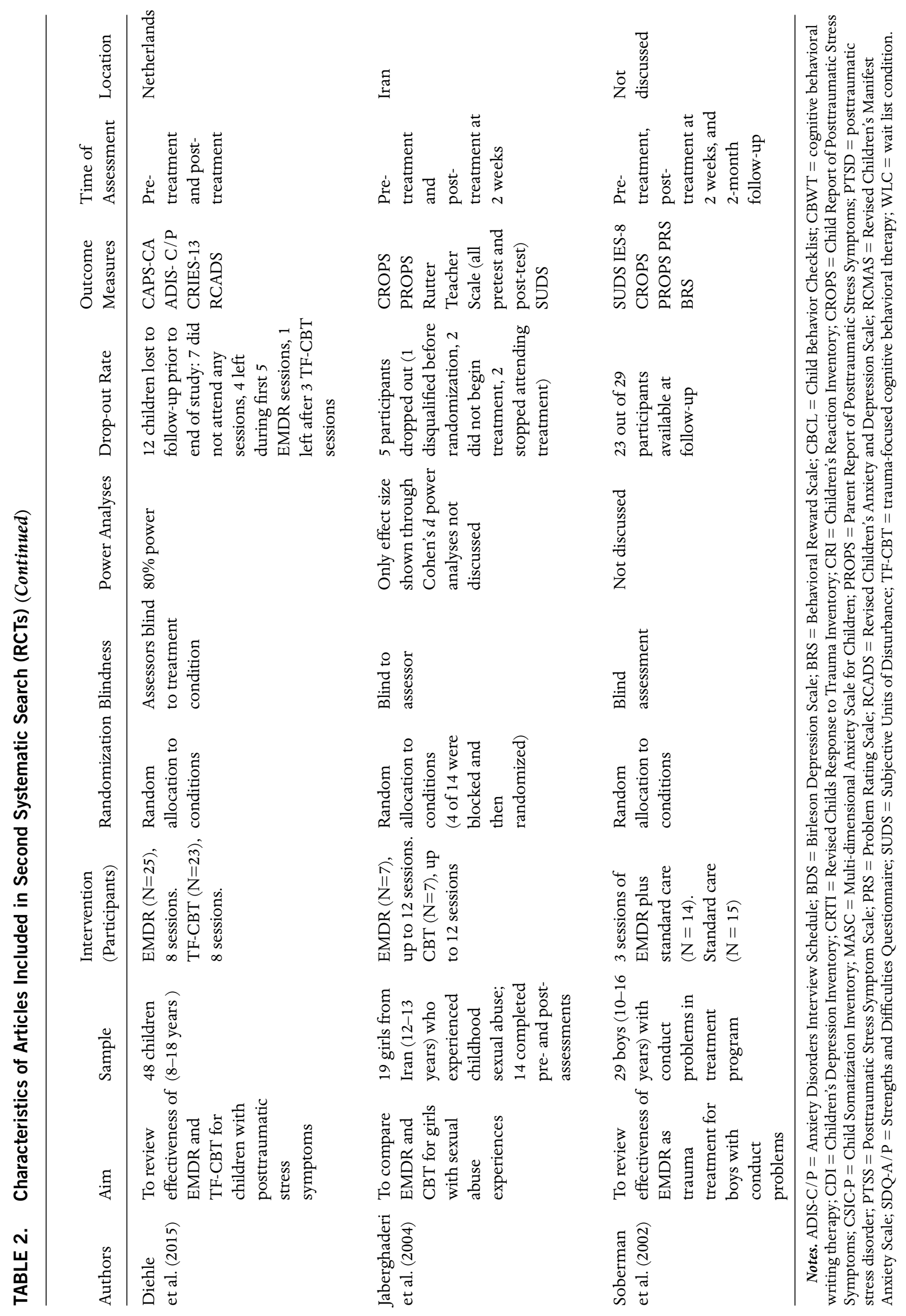




\section{Effect Sizes Reported in the RCTs}

Five studies reported effect sizes for EMDR. Diehle et al. (2015) found a medium effect size for EMDR ( $d$ $=0.72)$ compared to a large effect size for TF-CBT $(d=1.1)$. De Roos et al. $(2011,2017)$ identified substantial effect sizes found for PTSD on both EMDR $(d=1.27)$ and CBT $(d=1.24)$, with smaller effect sizes for anxiety, depression, and behavior problems $(d=$ .39-1.03). Finally, Jaberghaderi et al. (2004) reported that CBT and EMDR showed large effect sizes for PTSD and a medium effect size for positive behavior change. The largest effect size in the Ahmad and Sundelin-Wahlsten (2008) study was for reexperiencing symptoms $(d=.40)$, and smallest for the hyperarousal scale $(d=.01)$.

\section{Comparisons to Wait List and Standard Care}

Four studies compared EMDR to a wait-list condition or treatment as usual, and showed significant reductions in PTSD for children who received EMDR. Chemtob et al. (2002) found that in contrast to children who experienced EMDR, the wait-list condition showed no change in depression or anxiety from baseline to pretreatment; however, once treated, the wait-list group also showed change in all outcome measures similar to the initially treated groups. Likewise in Ahmad and Sundelin-Wahlsten (2008), children experienced greater symptom improvement with EMDR compared to the waitl ist group. De Roos et al. (2017) demonstrated large effect sizes for both cognitive behavioral writing therapy (CBWT) and EMDR versus the wait list condition for traumarelated appraisals, anxiety, depression, behavior problems, and decreases in trauma symptoms. This study reported impressive reductions in PTSD. Posttreatment, $92.5 \%$ of EMDR and $90.2 \%$ of CBWT participants no longer met PTSD criteria. Treatment gains were maintained at 3-month follow-up, and for EMDR participants at 12-months posttreatment, PTSD diagnostic remissions reached $100 \%$. There were no significant changes during wait-list periods. In an RCT, Soberman et al. (2002) compared the effectiveness of EMDR with standard care for boys, aged 10-16 years, with behavior problems. Boys who received EMDR experienced significant reductions in PTSD and behavior problems compared to the control group at 2 weeks posttreatment and at 2-month follow-up.

\section{Comparisons to TF-CBT}

Four studies compared EMDR to TF-CBT. Jaberghaderi et al. (2004) found large effect sizes for reduced PTSD for both EMDR and CBT with sexually abused girls aged 12-13 years from Iran. Both treatments also achieved a medium effect size for positive behavior change. Similarly, De Roos et al. (2011) and De Roos et al. (2017) found substantial and statistically significant reductions in PTSD for children who had experienced a single-event trauma. Both treatments also achieved comparable gains in reduced anxiety, depression, and behavior problems. In contrast, Diehle et al. (2015) found that although EMDR and CBT were effective in reducing PTSD for children aged 8-18 years, CBT achieved slightly greater gains.

\section{Types of Traumas}

The types of traumatic events which were successfully treated with EMDR included child sexual abuse (Jaberghaderi et al., 2004), disasters (Chemtob et al., 2002; De Roos et al., 2011), and single-event trauma experiences (De Roos et al., 2017).

\section{Effects for Comorbid Symptoms}

De Roos et al. (2017) examined the effectiveness of EMDR for single-incident trauma and found significant reductions in PTSD and comorbid symptoms in children/adolescents being treated for PTSD. The authors concluded both EMDR and CBWT are brief and effective therapies. De Roos et al. (2011) also found that children exposed to disaster benefitted from CBT and EMDR in that reductions were reported in PTSD symptoms, depression, anxiety, and problematic behavior. Chemtob et al. (2002) similarly found that children who presented with disaster-related PTSD and who received EMDR showed reduced scores on the Children's Reaction Inventory (CRI) as well as a reduced number of health visits to nurse. Chemtob et al. (2002) concluded EMDR appeared beneficial for children exposed to disaster and who present with PTSD symptoms (Chemtob et al., 2002). Jaberghaderi et al. (2004), in a study with Iranian girls who had been sexually abused, reported a statistically significant reduction in PTSD that equated to a large effect size $(d=2.0)$. Reductions were also reported for behavioral difficulties following EMDR. Both CBT and EMDR were found to be effective. Finally, Soberman et al. (2002) in assessing the effectiveness of EMDR for boys with conduct problems placed in residential units, found EMDR led to reduced PTSD and reduced memory-related distress, as well as fewer problem behaviors at 2-month follow-up.

Ahmad and Sundelin-Wahlsten (2008) and Diehle et al. (2015) demonstrated treatment effects on PTSD 
measures (Cohen's $d=0.41$ and 0.83 , respectively) when children (6-18yrs) who presented with PTSD from various and multiple trauma events (e.g., accidents, sexual assault, exposure to domestic violence), with comorbid symptom presentations, were treated with up to eight EMDR sessions. Ahmad and Sundelin-Wahlsten (2008) reported that of the 33 participants, $78 \%$ also had comorbid diagnoses, and results demonstrated no impact of comorbid diagnosis on treatment outcome. Diehle et al. (2015) reported that effect sizes for children who experienced singleor multiple-event trauma were about the same.

\section{Culture}

EMDR was also effective in reducing PTSD in differing locations and cultures. In Iran, Jaberghaderi et al. (2004) found statistical reductions in PTSD and behavior following EMDR and CBT. In Amsterdam (Diehle et al., 2015) and other parts of the Netherlands (De Roos et al., 2011, 2017), TF-CBT, CBWT, and EMDR were effective in reducing PTSD. Reduced PTSD and behavioral change were also reported in Hawaii (Chemtob et al., 2002).

\section{Sex}

Boys and girls experienced reductions in PTSD following EMDR. Most studies in the review included mixedgender participants except for Soberman et al., 2002 (boys); and Jaberghaderi et al., 2004 (Iranian girls with a sexual abuse history). In each study gains were made in reduced PTSD and behavior change; for example, Soberman et al. (2002) found EMDR as effective trauma treatment for boys with conduct problems.

\section{Maintenance of Gains}

Maintenance of gains (reduced PTSD) over different time periods has been demonstrated with EMDR. Follow-up time periods posttreatment include 2 weeks (Jaberghaderi et al., 2004), although this provides an insufficient timescale to assess whether gains were maintained in the long term; 2 months (Soberman et al., 2002) where the EMDR group showed reductions in problem behavior for boys with conduct problems at follow-up; 3 months (De Roos et al., 2011; De Roos et al., 2017); 6 months (Chemtob et al., 2002); and 12 months (De Roos et al., 2017). Overall effectiveness and reductions in PTSD, anxiety, depression, and behavioral symptoms were found to be maintained at follow-up.

\section{Discussion}

Contrary to NICE (2018), the current review identified that prior meta-analyses, systematic reviews, and RCTs for children and adolescents demonstrate that EMDR appears to be an efficacious treatment for children and adolescents with PTSD and other traumarelated symptoms. This finding is, therefore, in line with conclusions in international treatment guidelines for EMDR and PTSD (ISTSS Guidelines Committee, 2018; WHO, 2013). This is an important finding given the extent of trauma exposure for children globally, their developmental immaturity, and resultant symptoms in the short and long term (Yule et al., 2000). In addition to PTSD, the evidence indicates that EMDR may also be effective in reducing depression, anxiety, and behavioral problems.

Unfortunately, however, and in support of NICE (2018) conclusions, the current review found that most empirical studies of the efficacy of EMDR, apart from the most recent, tend to be of low quality and variable in methodology. The latter makes comparisons across studies uncertain. For example, numbers of participants ranged widely across studies, as did participants' ages. There was typically a lack of exploration of issues specific to differences between child and adolescent respondents. Variability also occurred in the number of sessions administered as well as the duration of sessions. Most studies failed to report on any work with families. While most studies assessed similar symptoms, a variety of standardized measures were used for assessment. Not all studies conducted follow-up assessments, and the timescale of assessments varied. Only a few studies provided effect sizes along with a lack of consistency in analyzing and reporting effect sizes. Finally, although dropout rates were mostly reported, insufficient information was provided to understand the reasons for differences across studies. As the NICE review highlighted, such methodological discrepancies raise questions of confidence in EMDR studies, which in turn generates doubt regarding the efficacy of EMDR therapy. As Dominguez and Lee (2019) argue, it is, therefore, essential that independent and rigorous empirical studies of EMDR efficacy are conducted.

\section{Summary}

Despite these methodological limitations it was possible to determine that girls and boys benefitted from EMDR, as well as children from a small range of countries including the Netherlands, Iran, and the United States (Hawaii), and one or two unnamed locations. 
The literature suggests that effective treatments for PTSD (i.e., EMDR, TF-CBT), have equivalent effect sizes (ISTSS, 2019; WHO, 2013). There is however, a possible trend in the child literature that EMDR achieves effective results in fewer sessions that other effective treatments, but this was not analyzed in this review. Importantly gains achieved by EMDR were maintained at a range of follow-up times, up to 12 months. In terms of trauma exposure, EMDR was successful with both single-event traumas (Chemtob et al., 2002; De Roos et al., 2011; De Roos et al., 2017) as well as children who developed PTSD from multiple trauma experiences (Soberman et al., 2002), and from different trauma experiences such as child sexual abuse (Jaberghaderi et al., 2004), disaster (Chemtob et al., 2002), and other various trauma events (i.e. Ahmad \& Sundelin-Wahlsten, 2008). In short, some compelling evidence supports the conclusion that EMDR is a therapy that can address the nature and extent of child trauma exposure and resultant PTSD.

\section{Limitations}

In the literature reviewed, there were methodological limitations of the studies. Small sample sizes, differing measures across studies focusing on different symptoms, a narrow range of countries and cultures reported, and a lack of multiple studies focused on similar populations with similar difficulties undermines the strength of evidence. An obvious omission in the literature is RCTs with children under the age of 4 years. EMDR with younger children may use an adapted protocol. The effect of the various adjustments to the standard EMDR protocol to the outcome of treatment of PTSD in children is unknown, and requires further study.

The current study, in phase two, focused only on RCTs, and demonstrated effects within other experimental and quasi-experimental studies could provide additional findings to support the use of EMDR to treat trauma symptoms in children. As with adult studies, there was a lack of homogeneity across a wide range of research design factors. There is little agreement regarding the standardized measures to use, and follow-up time measurement periods are inconsistent and do not go beyond 12 months.

\section{Recommendations for Practice}

EMDR therapy, as an efficacious treatment, should be made available for children and adolescents with PTSD and comorbid symptoms. Boys and girls should have access to treatment and EMDR should be available across cultures.

\section{Recommendations for Future Research}

More RCTs need to be conducted on the efficacy of EMDR therapy for children and adolescents with PTSD. Specifically, RCTs with children 4 years and younger are needed with particular attention toward making any form of therapy appropriate for the age and developmental stage of the child. What we have yet to understand is why EMDR is not effective for some clients. Also, more research is needed to examine the effectiveness in children and adolescents with multiple trauma histories and present with complex PTSD. What also has not been examined is the comparative effect of weekly versus more intensive treatment for PTSD in children and adolescents. Comparative studies are needed across a wide range of countries and cultures and more studies are needed comparing gender differences.

\section{Conclusion}

Studies consistently show that EMDR therapy is effective within a small number of sessions for children and adolescents with PTSD. Indications are that EMDR is effective with both boys and girls primarily in western cultures, although there is some evidence to suggest this may occur across cultures. Gains in reduced symptoms persist over 12-month follow-up. In line with NICE (2018), there is, however, a limited number of RCTs that have investigated EMDR therapy for the treatment of PTSD in children and adolescents. RCTs for children under 4 years are missing from the literature. It may be that the standard EMDR protocol cannot be easily used in children under 4 years. In addition, consideration to cultural nuances and the age of children (other than under 18) as a possible variable in the RCTs was not included.

\section{References}

Adler-Tapia, R., \& Settle, C. (2009). Evidence of the efficacy of EMDR with children and adolescents in individual psychotherapy: A review of the research published in peer-reviewed journals. Journal of EMDR Practice and Research, 3(4), 232-247. doi:10.1891/1933-3196.3.4.232

Ahmad, A., Bo, L., \& Sundelin-Wahlsten, V. (2007). EMDR treatment for children with PTSD: Results of a randomized controlled trial. Nordic Journal of Psychiatry, 61(5), 349-354. doi:10.1080/08039480701643464 
Ahmad, A., \& Sundelin-Wahlsten, V. (2008). Applying EMDR on children with PTSD. European Child and Adolescent Psychiatry, 17(3), 127-132. doi:10.1007/s00787007-0646-8

Beer, R. (2018). Efficacy of EMDR therapy for children with PTSD: A review of the literature. Journal of EMDR Practice and Research, 12(4), 177-195. doi:10.1891/19333196.12.4.177

Brown, R. C., Witt, A., Fegert, J. M., Keller, F., Rassenhofer, M., \& Plener, P. L. (2017). Psychosocial interventions for children and adolescents after man-made and natural disasters: A meta-analysis and systematic review. Psychological Medicine, 47, 1893-1905. doi:10.1017/S0033291717000496

Chemtob, C. M., Nakashima, J., \& Carlson, J. G. (2002). Brief treatment for elementary school children with disaster-related posttraumatic stress disorder: A field study. Journal of Clinical Psychology, 58(1), 99-112. doi:10.1002/jclp. 1131

Chen, R., Gillespie, A., Zhao, Y., Xi, Y., Ren, Y., \& McLean, L. (2018). The efficacy of eye movement desensitization and reprocessing in children and adults who have experienced complex childhood trauma: A systematic review of randomized controlled trials. Frontiers in Psychology, 9, 534. doi:10.3389/fpsyg.2018.00534

Cook, A. (2005). Complex trauma in children and adolescents. Psychiatric Annals, 35(5), 390-398. doi:10.3928/ 00485713-20050501-05

Critical Appraisal Program. (2017a). CASP systematic review checklist. Retrieved from https: / / casp-uk.net/wpcontent/uploads/2018/01/CASP-Systematic-ReviewChecklist_2018.pdf

Critical Appraisal Program. (2017b). CASP systematic review checklist. Retrieved from https: / / casp-uk.net/wpcontent/ uploads / 2018/01/CASP-Randomised-Control led-Trial-Checklist-2018.pdf

De Bellis, M. D., \& Zisk, A. (2014). The biological effects of childhood trauma. Child and Adolescent Psychiatric Clinics, 23(2), 185-222. doi:10.1016/j.chc.2014.01.002

De Roos, C., Greenwald, R., Den Hollander-Gijsman, M., Noorthoorn, E., Van Buuren, S., \& De Jongh, A. (2011). A randomised comparison of cognitive behavioural therapy (CBT) and eye movement desensitisation and reprocessing (EMDR) in disaster-exposed children. European Journal of Psychotraumatology, 2(1), 5694.

De Roos, C., Van der Oord, S., Zijlstra, B., Lucassen, S., Perrin, S., Emmelkamp, P., \& De Jongh, A. (2017). Comparison of eye movement desensitization and reprocessing therapy, cognitive behavioral writing therapy, and wait-list in pediatric posttraumatic stress disorder following single-incident trauma: A multicenter randomized clinical trial. Journal of Child Psychology and Psychiatry, 58(11), 1219-1228. Retrieved from https://doiorg.silk.library.umass.edu/10.1111/jcpp. 12768

Diehle, J., Opmeer, B. C., Boer, F., Mannarino, A. P., \& Lindauer, R. J. (2015). Trauma-focused cognitive behavioral therapy or eye movement desensitization and reprocessing: What works in children with posttraumatic stress symptoms? A randomized controlled trial. European Child and Adolescent Psychiatry, 24(2), 227-236. doi:10.1007/s00787-014-0572-5

Dominguez, S. K., \& Lee, C. W. (2019). Differences in international guidelines regarding EMDR for PTSD: Why they diverge and suggestions for future research. Journal of EMDR Practice and Research, 13(4), 00-00.

Foa, E. B., \& Meadows, E. A. (1997). Psychosocial treatments for posttraumatic stress disorder: A critical review. Annual Review of Psychology, 48, 449-480.

Greenwald, R. (1995). Applying eye movement desensitization and reprocessing (EMDR) to the treatment of traumatized children: Five case studies. Anxiety Disorders Practice Journal, 1, 83-97. doi:10.1037/ h0094423

Greenwald, R. (1999). Eye movement desensitization and reprocessing $(E M D R)$ in child and adolescent psychotherapy. Northvale, NJ: Jason Aronson.

Greyber, L. R., Dulmus, C. N., \& Cristalli, M. E. (2012). Eye movement desensitization reprocessing, posttraumatic stress disorder, and trauma: A review of randomized controlled trials with children and adolescents. Child and Adolescent Social Work Journal, 29(5), 409-425. doi:10.1007/s10560-012-0266-0

International Society for Traumatic Stress Studies. (2018). Posttraumatic Stress Disorder Prevention and Treatment Guidelines: Methodology and recommendations. https: / / www.istss.org/getattachment/TreatingTrauma/New-ISTSS-Prevention-and-TreatmentGuidelines/ISTSS_Prevention Treatment Guidelines_ FNL.pdf.aspx

Jaberghaderi, N., Greenwald, R., Rubin, A., Zand, S. O., \& Dolatabadi, S. (2004). A comparison of CBT and EMDR for sexually-abused Iranian girls. Clinical Psychology and Psychotherapy, 11, 358-368. doi:10.1002/cpp.395

Jonkman, C. S., Verlinden, E., Bolle, E. A., Boer, F., \& Lindauer, R. J. (2013). Traumatic stress symptomatology after child maltreatment and single traumatic events: Different profiles. Journal of Traumatic Stress, 26(2), 225232. doi: $10.1002 /$ jts. 21792

Khan, A. M., Dar, S., Ahmed, R., Bachu, R., Adnan, M., \& Kotapati, V. P. (2018). Cognitive behavioral therapy versus eye movement desensitization and reprocessing in patients with post-traumatic stress disorder: Systematic review and meta-analysis of randomized clinical trials. Cureus, 10(9), e3250. doi:10.7759/ cureus.3250

Lovett, J. (2007). Small wonders: Healing childhood trauma with EMDR. Riverside: Free Press.

Mendoza-Weitman, L. (1992). Case study. EMDR Network Newsletter, 2(1), 11-12. doi:10.1007 / BF02306581

Moreno-Alcazar, A., Treen, D., Valiente-Gomez, A., SioEroles, A., Perez, V., Amann, B. L., \& Radua, J. (2017). Efficacy of eye movement desensitization and reprocessing in children and adolescent with posttraumatic stress disorder: A meta-analysis of randomized controlled trials. Frontiers in Psychology, 8, 1750. doi:10.3389/fpsyg.2017.01750. 
Nader, K. (2003). Memory traces unbound. Trends in Neurosciences, 26(2), 65-72. doi:10.1016/S0166-2236(02) 00042-5

National Institute for Health and Care Excellence. (2018). Post-traumatic stress disorder. NG116. Management of PTSD in children, young people and adults (1.6.13). Retrieved from https: / / www.nice.org.uk/guidance/ng116/resources/ posttraumatic-stress-disorder-pdf-66141601777861

Rodenburg, R., Benjamin, A., De Roos, C., Meijer, A. M., \& Stams, G. J. (2009). Efficacy of EMDR in children: A meta-analysis. Clinical Psychology Review, 29, 599-606. doi:10.1016/j.cpr.2009.06.008

Shapiro, F. (1989). Efficacy of the eye movement desensitization procedure in the treatment of traumatic memories. Journal of Traumatic Stress, 2, 199-223. doi: $10.1002 /$ jts.2490020207

Shapiro, F. (1991). Eye movement desensitization and reprocessing procedure: From EMD to EMD/R: A new treatment model for anxiety and related traumata. The Behavior Therapist, 14, 133-135. Retrieved from https: / / emdria.omeka.net/items/show/17610

Shapiro, F. (2001). Eye movement desensitization and reprocessing (EMDR): Basic principles, protocols, and procedures (2nd ed.). New York, NY: Guilford Press.

Shapiro, F. (2018). Eye movement desensitization and reprocessing (EMDR): Basic principles, protocols, and procedures (3rd ed.). London, UK: Guilford Press.

Shapiro, F., Kaslow, F. W., \& Maxfield, L. (2007). Handbook of EMDR and family therapy processes (1. Aufl ed.). Hoboken, NJ: Wiley.

Soberman, G. B., Greenwald, R., \& Rule, D. L. (2002). A controlled study of eye movement desensitization and reprocessing (EMDR) for boys with conduct problems. Trauma and Juvenile Delinquency: Theory, Research, and Interventions, 6(1), 217-236. doi:10.1300/J146v06n01_11

Tinker, R. H., \& Wilson, S. A. (1999). Through the eyes of a child: EMDR with children. New York, NY: Norton.

Van der Kolk, B. A. (2005). Developmental trauma disorder. Toward a rational diagnosis for children with complex trauma histories. Psychiatric Annals, 35(5), 401-408i.e.

Wilson, G., Farrell, D., Barron, I., Hutchins, J., Whybrow, D., \& Kiernan, M. D. (2018). The use of eye-movement desensitization reprocessing therapy in treating post-traumatic stress disorder: A systematic narrative review. Frontiers in Psychology, 9, 923. doi:10.3389/fpsyg.2018.00923

World Health Organization. (2013). Guidelines for the management of conditions that are specifically related to stress. Geneva, Switzerland: Author.

Yule, W., Bolton, D., Udwin, O., Boyle, S., O’Ryan, D., \& Nurrish, J. (2000). The long-term psychological effects of a disaster experienced in adolescence: The incidence and course of PTSD. Journal of Child Psychology and Psychiatry, 41(4), 503-511. doi:10.1111/1469-7610.00635

Disclosure. SDS receives fees for providing EMDR trainings. The other authors have no relevant financial interest or affiliations with any commercial interests related to the subjects discussed within this article.

Correspondence regarding this article should be directed to Ian Barron, Director, Center for International Education, Montague House, College of Education, University of Massachusetts. E-mail: Ibarron@umass.edu 